\title{
Unified model of loop quantum gravity and matter
}

\author{
Rodolfo Gambini ${ }^{1}$, S. Jay Olson ${ }^{2}$ and Jorge Pullin ${ }^{2}$ \\ 1. Instituto de Física, Facultad de Ciencias, Iguá 4225, esq. Mataojo, Montevideo, Uruguay. \\ 2. Department of Physics and Astronomy, Louisiana State University, Baton Rouge, LA 70803-4001
}

(Dated: September 7th 2004)

\begin{abstract}
We reconsider the unified model of gravitation and Yang-Mills interactions proposed by Chakraborty and Peldán, in the light of recent formal developments in loop quantum gravity. In particular, we show that one can promote the Hamiltonian constraint of the unified model to a well defined anomaly-free quantum operator using the techniques introduced by Thiemann, at least for the Euclidean theory. The Lorentzian version of the model can be consistently constructed, but at the moment appears to yield a correct weak field theory only under restrictive assumptions, and its quantization appears problematic.
\end{abstract}

\section{INTRODUCTION}

The "quantum geometry" approach to quantum gravity (also known as "loop quantum gravity") is based on the philosophy that one wishes to understand in detail the difficulties that arise when quantizing general relativity. Within this context, the issue of unification with other interactions has not been considered too frequently. Chakraborty and Peldán [1] proposed some years ago one possibility for unification. Their proposal is based on the following observation. Consider first the constraints of general relativity written in terms of Ashtekar's new variables,

$$
\begin{aligned}
D_{a} \tilde{E}^{a i} & =0, \\
\tilde{E}_{i}^{a} F_{a b}^{i} & =0, \\
\tilde{E}_{i}^{a} \tilde{E}_{j}^{a} F_{a b}^{k} \epsilon^{i j k} & =0 .
\end{aligned}
$$

At the moment we are referring to the original formulation of Ashtekar's new variables, where the densitized triads $\tilde{E}^{a i}$ and the connection $A_{a}^{i}$ that defines the covariant derivative $D_{a}$ and the curvature $F_{a b}^{i}$ are in principle complex $S U(2)$ valued quantities, and one needs to impose reality conditions to recover ordinary real general relativity. Chakraborty and Peldán propose generalizing these equation by considering that the variables take values in a more general gauge group. For simplicity, let us consider such group to be $S U(N)$. Now, equations (12) are valid in general for any gauge group. The Hamiltonian constraint (3) requires some further elaboration. In particular one needs a suitable generalization of the invariant tensor $\epsilon_{i j k}$. The proposal consists in considering the following object,

$$
\epsilon_{i j k}(\tilde{E})=\frac{\tilde{E}_{i}^{a} \tilde{E}_{j}^{b} \tilde{E}_{k}^{c} \eta_{a b c}}{\operatorname{det} q}
$$

where by $\operatorname{det} q$ we mean the determinant of the spatial metric $q_{a b}$,

$$
(\operatorname{det} q) q^{a b}=\tilde{E}_{i}^{a} \tilde{E}_{i}^{b} .
$$

It is straightforward to show that when the gauge group is $S U(2)$ the above expression reduces to the usual LeviCivita invariant tensor, and the spatial metric corresponds to the usual definition. If one considers a more general gauge group, like $S U(N)$, the $\epsilon(\tilde{E})$ transforms covariantly under the group action, but in general will be a function of the triads. Also the "spatial metric" is clearly a generalization. The resulting theory is a different theory than general relativity.

Chakraborty and Peldán were able to show that if one uses the proposed invariant tensor in the definition of the Hamiltonian constraint there exist suitable limits in which the theory can, for given gauge groups, approximate general relativity coupled with a Yang-Mills field. They have also shown that the resulting constraint algebra is first class.

At the time this proposal was presented, there were important difficulties unresolved. In particular it was not clear how to implement in the theory reality conditions that would guarantee that the resulting theory was real general relativity coupled to fields. An alternative was to consider the theory in a Euclidean signature and then all variables were real. But there was no straightforward answer in the case of a Lorentzian signature. Moreover the Hamiltonian (31) is non-polynomial in the basic variables, which seemed a significant obstacle for quantization at the time.

New developments have taken place since this proposal. In particular, Thiemann 2] has shown how to deal with non-polynomial terms in the Hamiltonian constraint, including the ones that arise when one considers the theory formulated in terms of real variables. In this note we would like to show that these developments can be applied to promote the Hamiltonian constraint of the Chakraborty and Peldán proposal to a well defined quantum operator, at least for the Euclidean theory. 


\section{MAKING THE (EUCLIDEAN) HAMILTONIAN CONSTRAINT POLYNOMIAL}

In the context of usual quantum geometry, Thiemann noticed the following important classical identity between the basic fields of the theory,

$$
\frac{\tilde{E}_{i}^{a} \tilde{E}_{j}^{b}}{\sqrt{\operatorname{det} q}}=2\left\{A_{c}, V\right\}^{k} \epsilon_{i j k} \eta^{a b c}
$$

where $V$ is the volume,

$$
V=\int d^{3} x \sqrt{\tilde{E}_{i}^{a} \tilde{E}_{j}^{b} \tilde{E}_{k}^{c} \epsilon^{i j k} \eta_{a b c}}
$$

The importance of the identity introduced by Thiemann is that it allows to express a non-polynomial expression in terms of an expression involving a Poisson bracket and polynomial dependences, which can be quantized straightforwardly.

We now proceed to generalize the above expression using the ideas of Chakraborty and Peldán. We replace the $\epsilon$ by equation (4) and let the internal gauge group be different from $S U(2)$. The volume becomes,

$$
V=\int d^{3} x \sqrt[4]{\tilde{E}_{i}^{a} \tilde{E}_{i}^{d} \tilde{E}_{j}^{b} \tilde{E}_{j}^{e} \tilde{E}_{k}^{c} \tilde{E}_{k}^{f} \eta_{a b c} \eta_{d e f}}
$$

From here we can derive the identity,

$$
2\left\{A_{a}, V\right\}^{i}=\frac{\tilde{E}_{i}^{d} \tilde{E}_{j}^{b} \tilde{E}_{j}^{e} \tilde{E}_{k}^{c} \tilde{E}_{k}^{f} \eta_{a b c} \eta_{d e f}}{(\operatorname{det} q)^{3 / 2}} .
$$

Remarkably, we can use this latter identity to write the (Euclidean) Hamiltonian constraint in the generalized case as,

$$
H(N)=\int d^{3} x\left\{A_{c}, V\right\}^{k} F_{a b}^{k} \tilde{\eta}^{a b c},
$$

and surprisingly, the Hamiltonian takes the same form as the one Thiemann proposed for general relativity. From here on one could proceed to the quantization using the same tools Thiemann used in the usual gravitational case. The generalization is straightforward, one would consider wavefunctions that are given by spin networks of the appropriate gauge group. It is remarkable that the generalization proposed by Chakraborty and Peldán acquires such a simple form in terms of spin networks.

One element we need to verify is the fact that the volume operator we are using does not take the standard form. Can we be sure it is a well defined operator? The easiest way to see this is to look at the version of the proof of finiteness of the volume operator presented by Ashtekar and Lewandowski [3]. They use strip operators to represent the triads, that correspond to triads smeared along a two dimensional surface. When one acts with the triads on a spin network state one generates a contribution proportional to the Dirac delta integrated along the spin net. That integral, together with the two dimensional integral of the smearing, yields a finite result when integrating the three dimensional Dirac delta. The same argument would apply to the operator we are presenting here.

\section{THE REAL LORENTZIAN THEORY}

Up to now we considered the Hamiltonian constraint that would arise with real variables in the Euclidean theory. Let us analyze the Lorentzian case. Ashtekar introduced the new variables for canonical gravity by starting with the usual formulation in terms of (densitized) triads $\tilde{E}_{i}^{a}$ and the canonical momentum $K_{a}^{i} \equiv K_{a b} E^{a i}$ related to the extrinsic curvature $K_{a b}$. He then considers a canonical transformation given by $A_{a}^{i}=i K_{a}^{i}+\Gamma_{a}^{i}$ where $\Gamma_{a}^{i}$ is the spin connection compatible with the triad. Barbero [4] made explicit what would happen if one considered a more general canonical transformation $A_{a}^{i}=\beta K_{a}^{i}+\Gamma_{a}^{i}$ with $\beta$ a parameter usually referred to as the Immirzi parameter. The Gauss law and diffeomorphism constraint remain unchanged. The Hamiltonian constraint however, becomes,

$$
\begin{aligned}
H(N) & =H^{E}(N)+H^{\text {Lor }}(N) \\
H^{E}(N) & =\int d^{3} x N(x) \frac{\tilde{E}_{i}^{a} \tilde{E}_{j}^{b}}{\sqrt{\operatorname{detq}}} F_{a b k} \epsilon^{i j k} \\
H^{\text {Lor }}(N) & =-\int d^{3} x N(x) 2 \frac{\left(\beta^{2}+1\right)}{\beta^{2}} \frac{\tilde{E}_{i}^{[a} \tilde{E}_{j}^{b]}}{\sqrt{\operatorname{detq}}} K_{i}^{a} K_{j}^{b},
\end{aligned}
$$


where we have distinguished the piece $H^{E}(N)$ that by itself would be the Hamiltonian constraint of the Euclidean theory if the variables are real, or the Hamiltonian of the Lorentzian theory if $\beta=i$ and the extra piece $H^{\text {Lor }}$ that is needed to be included in order to have the Lorentzian theory with $\beta$ a real quantity.

The choice of $\beta=i$ makes the second term vanish, and this was the original motivation for choosing that value in the Ashtekar formulation. If one chooses $\beta$ a real number, one needs to deal with the second term. Thiemann also taught us how to do this. He starts by noting that the trace of the extrinsic curvature satisfies,

$$
K=-\left\{V, \int d^{3} x H^{E}(x)\right\}
$$

where $H^{E}(x)$ is the single-densitized Euclidean Hamiltonian constraint $H^{E}(N)=\int d^{3} x N(x) H^{E}(x)$ and then the extrinsic curvature with one triad index can be written as,

$$
K_{a}^{i}=\left\{A_{a}^{i}, K\right\} .
$$

Finally, the extra portion of the Hamiltonian that arises in the Lorentzian theory, can therefore be written as,

$$
H^{\mathrm{Lor}}(N)=-\int d^{3} x N(x) 2 \frac{\left(\beta^{2}+1\right)}{\beta^{2}}\left\{A_{a}^{i}, V\right\}\left\{A_{b}^{j}, K\right\}\left\{A_{c}^{k}, K\right\} \tilde{\eta}^{a b c} \epsilon_{i j k} .
$$

The question now arises of how to generalize all this to to $S U(N)$ using the Chakraborty and Peldán idea. The particular form of $H^{\text {Lor }}$ we just mentioned may be problematic to generalize to $S U(N)$, since it involves explicitly $\epsilon_{i j k}$. If one replaces this quantity using the dynamical epsilon of Chakraborty and Peldán, it is not clear that the resulting constraint will close the appropriate constraint algebra. If we ignore this point for just a second, it is worthwhile noting that the resulting object is likely to be quantizable (see section 3.4 of [5]), and given the general form of the expressions the techniques of [5] produce, the resulting quantum operator may satisfy the correct commutator algebra, at least operating on diffeomorphism invariant functions. An alternative to this is to write $H^{\text {Lor }}$ without using the Poisson bracket tricks directly in terms of $\tilde{E}_{i}^{a}$ and $K_{a}^{i}$, the extrinsic curvature. The latter can be computed in terms of the canonical variables. The resulting expression does not involve $\epsilon^{i j k}$ at all, so generalizing it to $S U(N)$ will produce an expression that classically still satisfies the correct Poisson algebra. However, the techniques of [5] may not help in its quantization since they involve the use of epsilons.

Unfortunately, in both choices one is further confronted with the problem of reproducing Einstein's theory coupled to Yang-Mills using the same assumptions as Chakraborty and Peldán, namely that one is considering weak fields living on a DeSitter background. Without further assumptions the extra portion of the Hamiltonian constraint will yield terms coupling to the extrinsic curvature. One can eliminate these terms and obtain Einstein-Yang-Mills theory by choosing a slicing in which the extrinsic curvature vanishes, but this appears rather unnatural. This difficulty is rather fundamental, since when one uses real Ashtekar variables the condition for the background to be DeSitter space is more complicated than in the Euclidean case, and it involves explicitly the extrinsic curvature.

Another outstanding issue with this attempt to unification is the introduction of Fermions. This is due to the fact that in the action for Fermionic fields the gauge group generators appear explicitly, and key identities of $S U(2)$ play a crucial role in the calculations that lead to showing that the constraint algebra closes. It is possible that a different approach, for instance using supersymmetry, could be used. The Ashtekar formulation has been generalized to supergravity [6], so an avenue of attack of this problem is available. Scalar matter has been included in the model successfully by Chakraborty and Peldán.

\section{SUMMARY}

Summarizing, we have shown that Thiemann's quantization technique can be applied to the Chakraborty-Peldán unified model yielding a well defined quantum theory at least for the Euclidean signature. It is remarkable that the Euclidean portion of the Hamiltonian constraint is exactly the same as in the usual case just acting on spin network states of a more general gauge group. The full Lorentzian theory however has a Hamiltonian constraint that is more complicated than the purely gravitational one and it is not clear how to naturally get a limit in which it yields the Einstein-Yang-Mills theory. If this difficulty could be overcome and the model could be made to include Fermions via supersymmetry it would become a viable, elegant and mathematically well define way of having a unified theory of quantum fundamental interactions. 


\section{ACKNOWLEDGMENTS}

We wish to thank Octavio Obregón for discussions. This work was supported by grant nsf-phy0244335 and funds from the Horace Hearne Jr. Laboratory for Theoretical Physics.

[1] S. Chakraborty and P. Peldan, Phys. Rev. Lett. 73, 1195 (1994) arXiv:gr-qc/9401028; Int. J. Mod. Phys. D 3, 695 (1994) arXiv:gr-qc/9403002.

[2] T. Thiemann, Class. Quant. Grav. 15, 839 (1998) arXiv:gr-qc/9606089.

[3] A. Ashtekar and J. Lewandowski, Adv. Theor. Math. Phys. 1, 388 (1998) arXiv:gr-qc/9711031.

[4] J. F. Barbero Phys. Rev. D 51, 5507 (1995) arXiv:gr-qc/9410014.

[5] T. Thiemann, Class. Quant. Grav. 15, 1281 (1998) arXiv:gr-qc/9705019.

[6] T. Jacobson, Class. Quant. Grav.5(1988)923; D. Armand-Ugon, R. Gambini, O. Obregon, J. Pullin, Nucl. Phys. B460 (1996) 615 arXiv:hep-th/9508036; L. Urrutia, in "Proceedings of the 5th Mexican Workshop of particles and fields" J. C. D'Olivo, A. Fernandez, M. Perez, editors, AIP Press, Woodbury, NY 1996 hep-th/9609010; H. Kunitomo and T. Sano Prog. Theor. Phys. Suppl. 114, 31 (1993); T. Sano and J. Shiraishi, Nucl. Phys. B410, 423 (1993) arXiv:hep-th/9211104; T. Kadoyoshi and S. Nojiri, Mod. Phys. Lett. A12, 1165 (1997) arXiv:hep-th/9703149; K. Ezawa, Prog. Theor. Phys. 95, 863 (1996) arXiv:hep-th/9511047; Y. Ling, J. Math. Phys. 43, 154 (2002) arXiv:hep-th/0009020; Y. Ling and L. Smolin,Nucl. Phys. B 601, 191 (2001) arXiv:hep-th/0003285 